\title{
TANDEM-X DSM UNCERTAINTY MEASURES AND DEMONSTRATIONS
}

\author{
Cristian Rossi, Thomas Fritz and Michael Eineder \\ German Aerospace Center (DLR), Remote Sensing Technology Institute, Wessling, Germany.
}

\begin{abstract}
SAR interferometry is a well-known technique to produce digital surface models (DSM). In the global scale, the quality of these models significantly increased with the use of bistatic systems as for the TanDEM-X mission. DSM uncertainty can be measured in two scales: a global and a local one. A global scale analysis involves measures for the complete take, characterizing the whole correctness with a single parameter. A local scale analysis enters instead in the single elevation model cell, evaluating the quality obtained by different terrains and media present in the take. A coherence analysis is a typical measure for InSAR models. Globally, a simple average gives an overall quality measure hint. Locally, it provides a measure of the DSM pixel height error. Unfortunately, this measure lacks in several aspects as not considering unwrapping errors and not being valid for certain types of scattering. In this paper, various strategies for assessing elevation accuracy methods and demonstrate the different capabilities of the bistatic TanDEM-X system are proposed for variegated terrain types.
\end{abstract} \section{pect}

Index Terms - TanDEM-X, uncertainty, DEM, slope, as-

\section{UNCERTAINTY ASSESSMENT STRATEGIES}

In the global scale, an algorithm has been proposed to detect areas affected by unwrapping errors by employing the radargrammetric shifts generated during the coregistration stage of an interferometric processor [1]. A coarse reference elevation model is generated using this information. Large-scale errors can be detected by appropriately comparing reference and interferometric phase. As additional benefit, the algorithm provides an estimate of the so called absolute phase offset, thus not requiring external models or ground control points for the geocoding step. The approach is well-suited for operational processing schemes, i.e. detecting models affected by errors which compromise the overall elevation accuracy.

A local scale uncertainty analysis is more difficult as requiring the knowledge of the complex mechanisms involved in the electromagnetic signal propagation and backscattering. A straightforward way to check inaccurate model areas is to compare them with reference data. Root Mean Square Error (RMSE) and other statistical parameters can be easily computed, providing measures at several scales. Geo-spatial measures as variograms and Fourier transforms are additional tools for checking the spatial autocorrelation and anisotropy of the DSM error [2]. When reference data is not available, uncertainty can be investigated with strategies which depend on the local terrain type. For instance, geometrical distortions play a big role for urban and complex terrain models and one can expect inaccurate models over layover and shadow areas. In-processing techniques aimed to find distortions are useful to detect inaccuracies [3]. For moderate to smooth slopes, a combination of interferometric coherence and global error detectors (as aforementioned) provides a good uncertainty measure. Finally, when a temporal stack of models over a specific area is available, differential techniques help to discover relative errors or discrepancies due to temporal terrain variations, as for agricultural fields.

All these approaches are discussed in the next section with exemplary demonstrations.

\section{UNCERTAINTY DEMONSTRATIONS}

SAR stripmap data allows the generation of models with about 12 meters posting with HRTI-3 quality standards [4]. In fact, different accuracy levels can be awaited depending on the actual terrain to model. In particular, limitations have to be expected depending on the terrain slope and the medium to image. To demonstrate the differences in accuracy levels, three scenarios are considered: flat agricultural areas, moderate to complex terrain with various land covers and urban areas.

On the first side, it has been demonstrated how accuracy level is very high when mapping agricultural crops, even allowing volumetric change studies when employing temporal data stacks [5]. For this purpose, a test site widely employed for rice production in Turkey has been investigated with 16 dual-pol TanDEM-X acquisitions spanning two years and a dedicated ground campaign. The research demonstrated that for elevation accuracy studies at X-band wave polarization is not particularly relevant - only small elevation discrepancies around $8 \mathrm{~cm}$ were measured in dry season [6]. The increasing temporal elevation trend is well derived by TanDEM-X, with a general underestimation especially in the reproductive plant stage due to the wave interaction with lower portions of the canopy. Uncertainty measured by RMSE is contained 


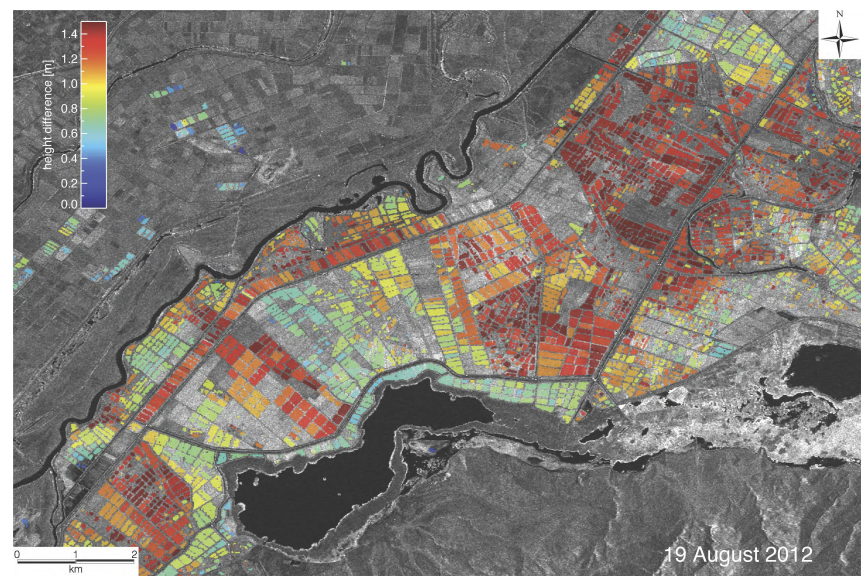

(a) Rice paddies elevation for a summer take. Crops show different growing.

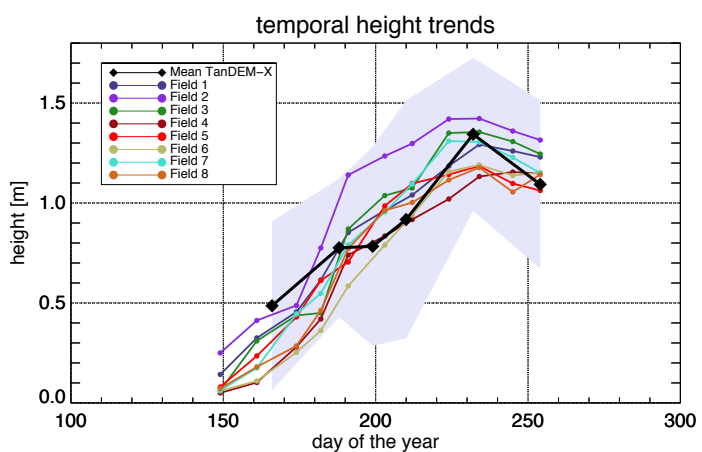

(b) Temporal mean TanDEM-X and reference fields heights. The variance of the TanDEM-X estimation is superimposed in violet.

Fig. 1. TanDEM-X smooth terrain exemplary surface model and uncertainty measure.

between few centimeters and about $20 \mathrm{~cm}$, depending on the actual phenological phase. In Fig. 1, the surface model (Fig. 1(a)) showing the different growing of the fields for a summer date is depicted. The overall mean trend is well fitting with reference fields (Fig. 1(b)). Accuracy is measured for this scenario with comparison with ground truth coming from a dedicated campaign.

On the other side, large limitations are measured for complex terrains. Besides the inability to map certain slopes due to the side-looking sensor geometry and the inherent geometrical distortions, unwrapping errors are most probable to appear and can be detected with the global indicator briefly described in the previous section. A test site in Spain, around the city of Terrassa, is chosen due to its variety and complexity. Height of ambiguity and looking angle at scene center are

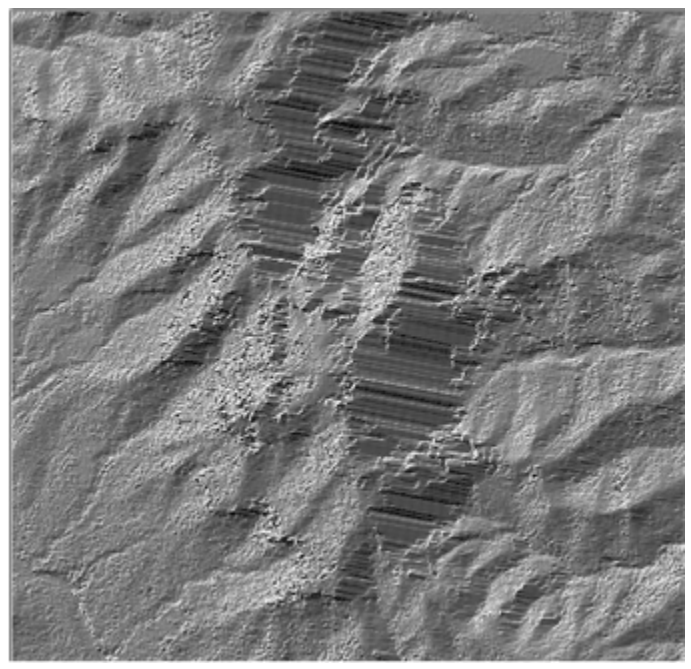

(a) Shaded raw DEM over cliffs and very steep slopes.

DEM slope and aspect distribution

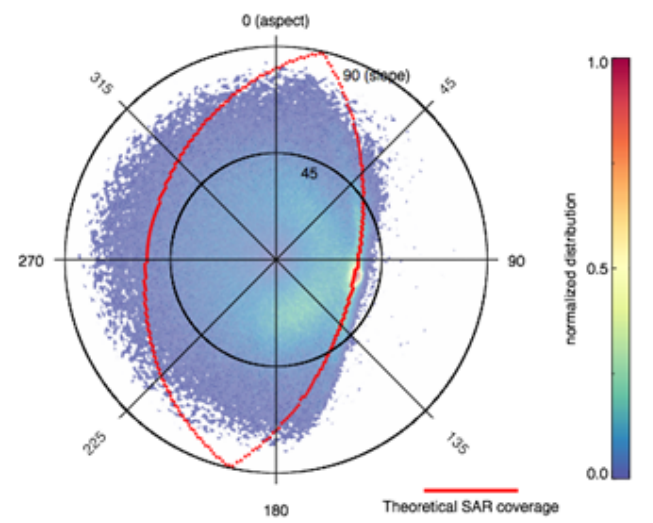

(b) DSM slope and aspect distribution plot.

Fig. 2. TanDEM-X complex terrain exemplary surface model and uncertainty measure, descending geometry.

$48 \mathrm{~m}$ and $33.6 \mathrm{deg}$ respectively. A crop of 4 by $4 \mathrm{~km}$ of the shaded raw DEM over cliffs and very steep slopes is showed in Fig. 2(a). The artificial slopes in the middle are caused by the layover effect [3]. The measured RMSE for the whole crop is about $12 \mathrm{~m}$. The difference with the previous scenario is very high. Next to RMSE, computed with LiDAR surface model, another accuracy instrument is the slope-visibility plot given any aspect angle. The plot provides the geographical angles visible by the sensor without geometric distortions. It is then useful to check invalid pixels given any DSM slope and aspect distribution and can be used for fusion result accuracy checks with other geometries [7]. In this plot, depicted in Fig. 2(b), the layover area is recognizable as the red spot at the border of the theoretical SAR distribution.

The same kind of analysis is peformed for moderate 


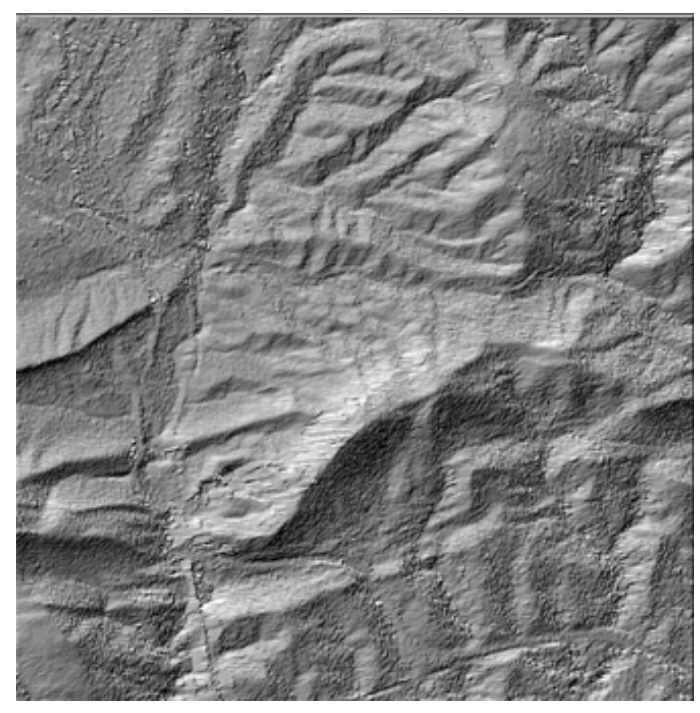

(a) Shaded raw DEM over moderate slopes.

DEM slope and aspect distribution

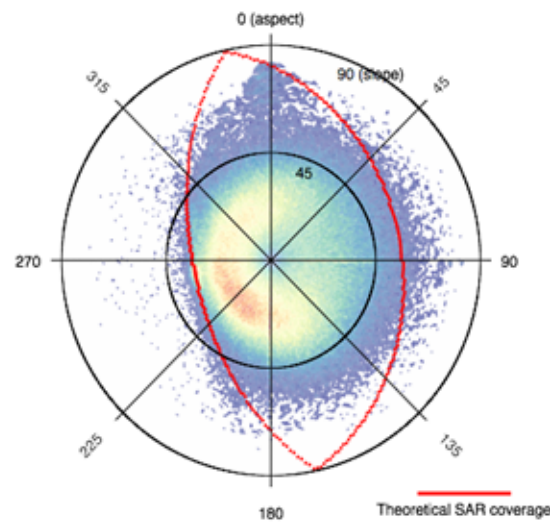

(b) DSM slope and aspect distribution plot.

Fig. 3. TanDEM-X moderate terrain exemplary surface model and uncertainty measure, ascending geometry.

slopes, smaller than $20 \mathrm{deg}$, and shown in Fig. 3. The measured accuracy is higher than the previous case, with a measured RMSE of about 4 meters. The accuracy of the model for aspect and slope angles is shown in Fig. 4 with histograms. Each bin represents the average error within the bin angles. Error dependency with aspect angle reveals two peaks around ground range angles. They represent facets where layover and shadow have the strongest impact. Average absolute error is contained within few meters - taking into account the bin size of $10 \mathrm{deg}$. Error dependency with slope shows an increasing error at increasing slopes. High slopes in this plot are characterized by a few outliers. Here, slopes larger than the looking angle, $34 \mathrm{deg}$, are fully under layover, so that their elevation results inaccurate as resem-

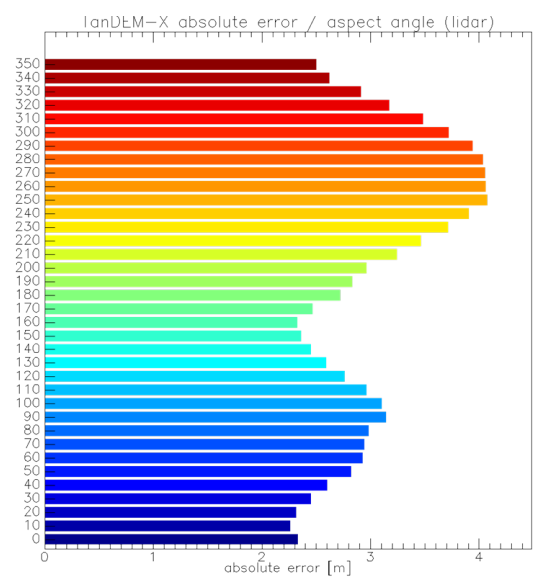

(a) TanDEM-X absolute error dependency with aspect angle. The two peaks correspond to layover and shadow areas.

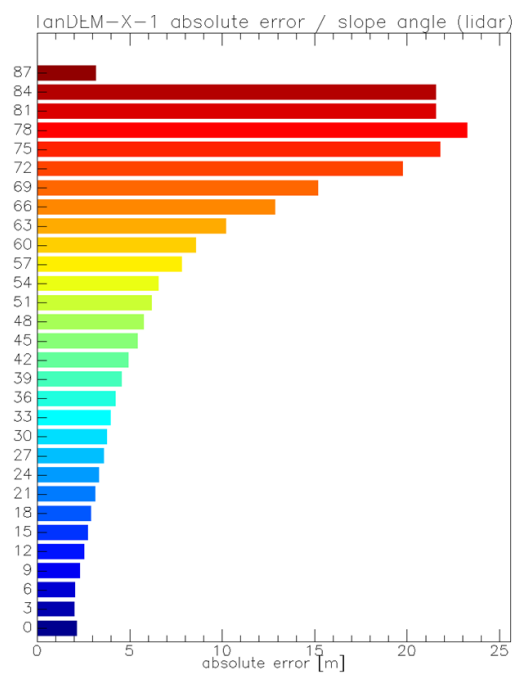

(b) TanDEM-X absolute error dependency with terrain slope. Slopes larger than $81 \mathrm{deg}$ are characterized by a few outliers and are not representative.

Fig. 4. TanDEM-X absolute error dependency with topographical angles. A LiDAR model is taken as reference for the computation.

bling multiple terrain portions. Geo-spatial analysis is also useful to measure uncertainty. Error spatial autocorrelation parameters are outcomes of a semi-variogram analysis, by checking the spatial dependence of a point with its neighbor. For the current test, a semi-variogram analysis estimates a spatially independent variance, i.e. nugget variance, of about $5 \mathrm{~m}$; a maximum semivariance, i.e. sill, of about $17 \mathrm{~m}$ and the distance for which ground resolution elements are not related, i.e. range, of about $6 \mathrm{~m}$.

Finally, the focus is posed on urban scenarios. Test-case is in this case the city of Berlin, Germany. The study is performed on several resolutions and processing parameters. For 


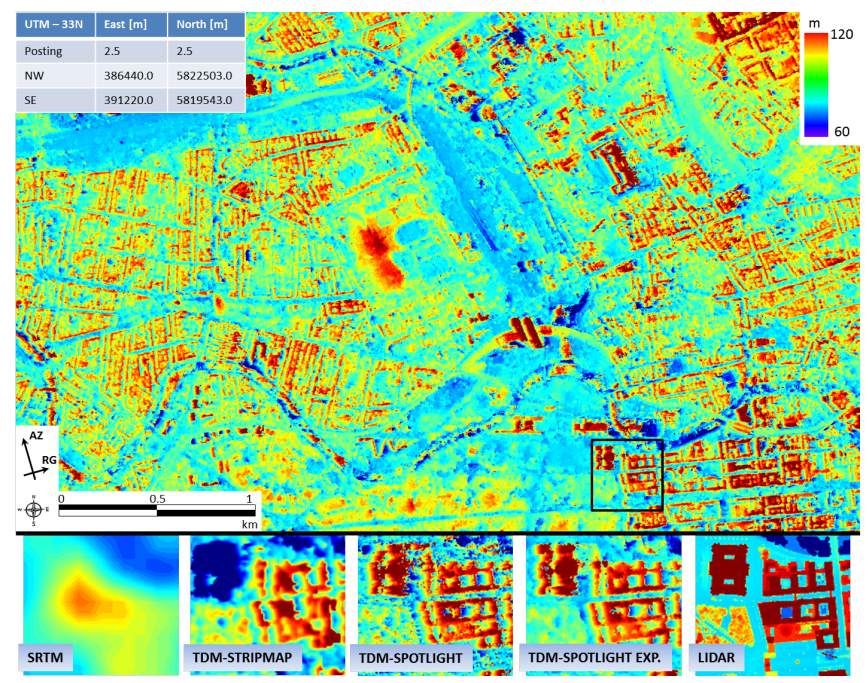

Fig. 5. TanDEM-X digital surface model of the city of Berlin (Germany) generated with high-resolution spotlight data. In the bottom, a zoom over reference buildings shows the different accuracy retrievable for various elevation models.

instance, locally focusing on roof height uncertainty, an adaptive processing yields height variance of $0.56 \mathrm{~m}$, whereas a standard processing of $4.72 \mathrm{~m}$ for five benchmark buildings [8]. The overall elevation RMSE between a $2.5 \mathrm{~m}$ rasterized DSM and a reference is about $8 \mathrm{~m}$ for the complete model shown in Fig. 5. The derived accuracy level places urban configuration in the middle between easy and complex terrain. Actually, the interest may be on the single structure height reconstruction more than on the overall quality, and the study is driven on the layover portion of buildings. It is shown how layover strongly affects the model accuracy, by creating artificial elevation ramps [3]. Detection of layover is then a fundamental step. Here, the uncertainty assessment strategy moves to firstly localize problematic regions and then to assess the error over there. Mean RMSE error for all the detected buildings results about 9 meters.

\section{CONCLUSIONS}

This paper provides a concise description of developed uncertainty measures and tests the accuracy of TanDEM-X models, generated with the Integrated TanDEM-X Processor, for some terrain samples. It has been demonstrated how model accuracy depends on the terrain to be mapped and on the topographical features. First of all, the measured elevation is the scattering phase center. This may lie inside the medium to be imaged, thus yielding height underestimations when compared to other sources. The demonstration performed for rice canopies revealed a little penetration in X-band, allowing the tracking of phenonological stages. Second, terrain slope strongly affects the final model quality. Geometrical decorrelations impact has been tested for mountanous areas and for urban scenarios. Model trend has been predicted in case of layover. Among uncertainty evaluation instruments, effective in-processing inaccurate area markers have been demonstrated to be the phase unwrapping error detector and the layover mask. Post-processing evaluations usually require a reference. A focus can be on the correlation of the error, as shortly seen for smooth terrain, or on a global absolute error evaluation with the RMSE. This last parameter is the reference one to have a direct evaluation. RMSE derived for the proposed experiments are $0.2 \mathrm{~m}, 4 \mathrm{~m}, 8 \mathrm{~m}$ and $12 \mathrm{~m}$ for very smooth terrain, moderate slope terrain, urban areas (at higher resolution) and high slope terrain respectively.

\section{REFERENCES}

[1] C. Rossi, et al., "TanDEM-X calibrated raw DEM generation," ISPRS Journal of Photogrammetry and Remote Sensing, vol. 73, pp. 12-20, 2012.

[2] L. Hongxing and K. C. Jezek, "Investigating DEM error patterns by directional variograms and Fourier analysis,", Geographical Analysis, vol. 31, pp. 249-266, 1999.

[3] C. Rossi and M. Eineder, "High-resolution InSAR building layovers detection and exploitation," IEEE Trans. Geosci. Remote Sens., to be published.

[4] G. Krieger, et al. "TanDEM-X: A satellite formation for high-resolution SAR interferometry," IEEE Trans. Geosci. Remote Sens., vol. 45, pp. 3317-3341, 2007.

[5] C. Rossi and E. Erten, "Paddy-rice monitoring using TanDEM-X," IEEE Trans. Geosci. Remote Sens., vol. 53, pp. 900-910, 2015.

[6] E. Erten, et al., "Polarization Impact in TanDEM-X Data Over Vertical-Oriented Vegetation: The PaddyRice Case Study," IEEE Geosci. Remote Sens. Lett., 2015.

[7] R. Deo, et al., "Framework for Fusion of Ascending and Descending Pass TanDEM-X Raw DEMs," IEEE J. Sel. Topics Appl. Earth Observ. Remote Sens., 2015.

[8] C. Rossi and S. Gernhardt, "Urban DEM Generation, Analysis and Enhancements using TanDEM-X," ISPRS Journal of Photogrammetry and Remote Sensing, vol. 85, pp. 120-131, 2013. 